\title{
A Frequency Comb Transferred by Surface Plasmon Resonance
}

\author{
Xiao Tao Geng ${ }^{1,2}$, Young-Jin Kim ${ }^{3}$, Seungchul Kim ${ }^{1,2}$, Dong Eon Kim ${ }^{1,2}$ \\ ${ }^{1}$ Max Planck Center for Attosecond Science \\ Max Planck Pohang University of Science and Technology, Korea Res. Initiative, \\ Pohang, Gyeongbuk 376-73, South Korea \\ ${ }^{2}$ Department of Physics, Center for Attosecond Science and Technology, Pohang University of Science and Technology \\ Pohang, Gyeongbuk 376-73, South Korea \\ ${ }^{3}$ School of Mechanical and Aerospace Engineering, Nanyang Technological University \\ 50 Nanyang Avenue, Singapore 639798, Singapore \\ xtgeng@ postech.ac.kr; yj.kim@ntu.edu.sg; inter99@postech.ac.kr; kimd@ postech.ac.kr
}

\section{Extended Abstract}

Frequency comb composed by millions of narrow linewidth optical modes referenced to an atomic clock has shown its remarkable potentials in time/frequency metrology and atomic/molecular spectroscopy ${ }^{1}$. It is still newly expanding its application area to nonlinear spectroscopy as demonstrated in coherent Raman spectroscopy and to coherent quantum metrology for entangled atomic qubits. Yet, the correlation of absolute optical comb with surface plasmon remains unexplored, although plasmonics are already played important roles in such fields by manipulating the light within a subwavelength scale. We report that optical frequency comb can be exactly transformed into plasmonic comb at metallic nanostructures with a constant frequency spacing of $75 \mathrm{MHz}$ and linewidth of $\sim 1 \mathrm{~Hz}$, equal to stabilized optical comb ${ }^{2}$. It reverted to original comb modes vice versa without noticeable degradation. These intriguing results imply that welldefined frequency comb is ready to be applied to various nanoplasmonic spectroscopy, coherent quantum metrology, and other nano-photonics with its superior performances.

Coupling surface plasmons (SPs), collective charge oscillations produced by the resonant interaction of light and free electrons on the interface of metallic and dielectric materials, to frequency comb creates numerous advantages. First, SP can allow for the frequency comb to access nanoscopic volumes that surpass the diffraction limit. Second, the field enhancement by localized SP enables the highly sensitive detection of weak signals, even from a single molecule (for example, surface-enhanced Raman scattering). Third, next-generation photonic devices and circuits can be implemented within a small subwavelength volume by all-optical control of light properties (amplitude, phase and polarization state) in plasmonic nanostructures within ultrafast time scales. However, the superior performance of the frequency comb, such as absolute frequency uncertainty, high-frequency stability and narrow linewidth, could deteriorate during the photonplasmon conversion process. For exploring novel combination of frequency comb and SP resonance, it is prerequisite to verify that frequency comb maintains its performance under plasmonic resonance; however, there have been no studies to date. In the following, we report that frequency comb successfully maintains core performances in photon-plasmon conversion by exploiting plasmonic extraordinary transmission through a subwavelength plasmonic hole array. This implies that the original frequency comb can be transformed into a form of plasmonic comb on metallic nanostructures and reverted to an original frequency comb without noticeable degradation in absolute frequency position, stability and linewidth. The superior performance of well-defined frequency combs can therefore be applied to various nanoplasmonic spectroscopy, coherent quantum metrology and subwavelength photonic circuits.

In this work, we have studied SP resonance effects on frequency comb structure in the plasmonic EOT of light through a subwavelength metallic nano hole $a_{r r a y}{ }^{2}$. The frequency comb was transduced to plasmonic mode in the sample and reverted to photonic mode without significant changes in linewidth, frequency shift, $\mathrm{S} / \mathrm{N}$ ratio, phase noise and Allan deviation. The linewidth broadening was $\sim 1 \mathrm{~Hz}$ (instrument limited), frequency inaccuracy was $6.51 \times 10^{-19}, \mathrm{~S} / \mathrm{N}$ ratio was higher than $60 \mathrm{~dB}$, Allan deviation increased by $2.92 \times 10^{-19}$ at $100 \mathrm{~s}$ averaging time. This outstanding frequency comb performance in plasmonic nanostructures enables a highly sensitive, high accurate and broadband measurement with direct traceability to standards. This inclusion of frequency comb has the potential to accelerate progresses in various plasmonic 
applications such as bio-chemical spectroscopy or sensing, quantum optics and sub-diffraction limit biomedicalimaging. With the aid of SP, frequency-comb referenced high-speed coherent anti-stokes Raman spectroscopy ${ }^{3}$ can be implemented in much smaller nanoscopic volume being requested for single-molecule detection, for example, surfaceenhanced coherent anti-stokes Raman spectroscopy.

\section{References}

[1] T. W. Hänsch, "Nobel Lecture: Passion for precision,” Rev. Mod. Phys., vol. 78, pp. 1297-1309, 2006.

[2] X. T. Geng, "Frequency comb transferred by surface plasmon resonance," Nature communications, vol. 7, pp. 10685, 2016.

[3] T. Ideguchi, et al., "Coherent Raman spectro-imaging with laser frequency combs." Nature, vol. 502, pp. 355-358, 2013. 